\title{
Ligas acadêmicas de Odontologia: uma revisão de literatura
}

\author{
Tamires Santos de Melo*; Maria Cardoso Berry**; Maria Isabel Souza***
}

\author{
* Graduanda em Odontologia, Faculdade de Odontologia, \\ UERJ \\ ** Pós-graduada em Odontopediatria, Faculdade de \\ Odontologia, UFRJ, Mestre em Telessaúde, Faculdade de \\ Medicina, UERJ \\ *** Professora Associada da Faculdade de Odontologia, UERJ
}

Recebido em 16/03/2018. Aprovado em 07/01/2019.

\begin{abstract}
RESUMO
Ligas acadêmicas são definidas como atividades extracurriculares coordenadas por estudantes com a tutoria e participação de docentes, tendo também a participação de pesquisadores e profissionais de saúde. Os integrantes almejam contribuir com suas experiências, explorar e aprofundar o conhecimento sobre um determinado tema. O presente estudo tem por objetivo buscar na literatura subsídios para delinear a importância e contribuição das ligas acadêmicas para a formação dos discentes em Odontologia. Na estratégia de busca foram utilizados os descritores "Educação"; "Educação em saúde"; "Atividade extracurricular"; "Ligas Acadêmicas"; e "Odontologia", nos idiomas inglês, português e espanhol, nas bases de dados PubMed, Google Scholar, MEDLINE, LILACS e SciELO. Foram encontrados 3.552 artigos científicos publicados de 2000 a 2017. Após a aplicação dos critérios de inclusão e exclusão foram selecionados 30 documentos, os quais foram consultados integralmente. Os resultados encontrados foram sistematizados em 4 eixos temáticos: Saúde, Odontologia, Atividade extracurricular e Impacto na Formação Acadêmica. Com base na literatura consultada, conclui-se que apesar da quantidade expressiva de artigos encontrados, poucos abordam especificamente o tema Liga Acadêmica. Os artigos específicos ressaltam que as ligas acadêmicas são benéficas para seus participantes, aperfeiçoando a prática de autoaprendizado, liderança, inter-relação, empatia e a troca mútua de conhecimento entre seus participantes e sociedade.
\end{abstract}

Descritores: Educação. Educação em Saúde. Odontologia.

\section{INTRODUÇÃO}

A ideia de uma liga acadêmica não é algo novo. Durante os anos 1920 foi criada a primeira
Liga Acadêmica (LA), nomeada de Liga Acadêmica de Combate à Sífilis, na Faculdade de Medicina de Ribeirão Preto-USP ${ }^{1,2}$. Ao longo dos 
anos foram surgindo outras iniciativas semelhantes a esta nas faculdades de Medicina do Brasil, como por exemplo a Liga de Combate à Hanseníase Luiz Marino Bechelli, fundada em 1991. No período da ditadura militar, as ligas acadêmicas tinham como meta interpelar as formas de ensino universitário da época, tal qual o desfecho que levaria a ascensão técnico-científica em curso ${ }^{3}$.

Na década de 90, visando sanar carências curriculares e promover mudanças, as faculdades de Medicina iniciaram processos de reforma curricular, pautando-se em três pontos fundamentais: educação, pesquisa e extensãoassistência ${ }^{4}$.

Com base nesses alicerces, as ligas acadêmicas passaram a ter um protagonismo maior, promovendo atividades com o objetivo de preencher as lacunas existentes na formação dos alunos de graduação bem como para a adaptação dos currículos a estas novas mudanças ${ }^{5,6}$.

O uso crescente dessa ferramenta educacional teve contribuição das Diretrizes Curriculares Nacionais (DCN), resolução CNE/CES de 2010, que recomendam a realização de atividades complementares que contribuam para o ganho de conhecimento do estudante, de forma independente, sendo presencial ou à distância ${ }^{7}$. Assim sendo, as ligas acadêmica de Medicina de Família e Comunidade baseiam suas ações nas DCN para graduação de Medicina durante a execução de funções na Atenção Primária à Saúde ${ }^{8}$.

A LA apresenta algumas vantagens como: estimular a iniciativa para auto-aprendizagem e senso crítico, preparar os acadêmicos frente aos avanços do conhecimento cientifico, familiarização precoce com os fatores que contribuem e permeiam a relação de saúde-doença, possibilitando ao acadêmico compreender e observar as reais necessidades da comunidade local e a inter-relação entre indivíduos de diferentes estágios da profissão, e possibilita contato entre calouros e veteranos ${ }^{9}$.
As LAs são constituídas por atividades coordenadas por estudantes de diferentes períodos da graduação, com a participação de docentes, pesquisadores e profissionais de saúde que desejam contribuir com suas experiências, explorar e aprofundar um tema ${ }^{10,11}$. As LAs podem ter diferentes formas, porém devem apresentar regras claras e bem definidas para então servir de guia na sua constituição.

Dentre as atividades propostas por uma liga, encontram-se aulas teóricas, cursos, hands on, simpósios e congressos, elaboração de projetos de pesquisa, campanhas visando assistência de saúde em diversos locais e eventos públicos de promoção à saúde ${ }^{12,13}$.

As LAs promovem benefícios tanto para o acadêmico como também para a comunidade. Tratando-se em especial da área de saúde, existem relatos que expõem as contribuições efetivas da Liga de Educação em Saúde (LES) como a existência de espaço para debates e articulação de promoção de saúde, buscando trabalhar intimamente com a comunidade e entender as interações entre os saberes científicos e populares, contribuindo para o aprimoramento do conhecimento em saúde ${ }^{14}$.

Outro exemplo que enfatiza a importância das LAs é o relatado feito pela Liga Acadêmica de Psicologia Escolar (LAPES), vinculada a Escola Baiana de Medicina e Saúde Pública, criada com o objetivo de desenvolver um espaço para discussões sobre Psicologia no contexto escolar, além de aplicar o conhecimento construído nas práticas de intervenção mais adequada no desenvolvimento pedagógico e social de cada individuo abordado. Esta liga, semestralmente, seleciona temas, tendo como base o tripé da universidade. Para além dessa vasta experiência que o acadêmico tem ao participar da LAPES, anualmente, são feitas reuniões com o tema "As Práticas do (a) Psicólogo (a) na Escola" onde dois profissionais da área de psicologia expõem suas experiências no âmbito 
escolar $^{15}$.

Em relação à Odontologia, é possível observar grandes contribuições por parte das LAs, levando em consideração os pontos positivos já mencionados para formação acadêmica. Atualmente, no Brasil, a área odontológica apresenta vasto conhecimento científicotecnológico, porém, ao mesmo tempo em que isso ocorre, temos uma grande parte da população sem renda para custear o tratamento odontológico. Sendo assim, o resultado encontrado é um grande número de pessoas com problemas de saúde bucal e geral. Portanto, é necessário que os graduandos entendam este contexto social e pensem em estratégias efetivas para o cenário precário de saúde geral e bucal. Essas competências são preconizadas pelas DCN de Odontologia, que enfatizam a importância de formar um profissional com perfil crítico, humanista, reflexivo e generalista ${ }^{16}$.

Contudo, vale ressaltar que os currículos tradicionais dos cursos de Odontologia nem sempre enfatizam ou desenvolvem esse tipo de aptidão nos seus graduandos, tornando as LAs um recurso importante e fornecendo aos estudantes um campo que lhes possibilita debates, experiências com pacientes/comunidade já nos primeiros anos do curso, contato precoce com especialistas e vivência com realidades diversas de saúde, além de favorecer a aproximação da universidade com a comunidade $^{17,18}$.

A aproximação entre universidade $\mathrm{e}$ estudantes pode ser observada no relato de caso da Liga Acadêmica Baiana de Educação em Saúde Bucal (LABESB), onde foram expostas suas experiências de 2011 a 2013, por meio da execução de 32 atividades educativas em comunidades de Salvador/BA, que envolveram diversos grupos etários (de bebês até idosos) em suas ações. O principal objetivo do trabalho era conscientizar e estimular os pacientes a mudar suas posturas em relação à sua própria saúde. Juntamente a essas atividades, realizaram instruções e atividade de higiene oral supervisionada. Essas ações não se limitaram à capital, a LABESB realizou também idas ao interior com intuito de promover saúde em outras comunidades, além de contribuir permanentemente com trabalhos educativos em saúde no Centro de Integração Familiar (CEIFAR - Instituição civil filantrópica fundada na década de 90) ${ }^{16}$.

Tendo em vista o relevante papel das LAs como ferramenta educacional e conhecimento científico, o objetivo da presente revisão foi buscar dentro da literatura disponível subsídios para delinear a importância e contribuição das LAs na formação de estudantes de graduação na área de Odontologia, bem como orientar o processos para desenvolver deste tipo de atividade nas instituições de ensino.

\section{METODOLOGIA}

A estratégia de busca na literatura utilizou a combinação dos descritores "Educação", "Educação em Saúde", "Atividades extracurriculares", "Ligas Acadêmicas" e "Odontologia", nos idiomas inglês, português e espanhol.

Os dados foram coletados nas bases PubMed, Google Scholar, MEDLINE, LILACS e SciELO. Os critérios de inclusão utilizados foram: acesso ao texto completo, publicação entre os anos 2000 e 2017, assim como descrição de relato de experiências e/ou aspectos educacionais na formação profissional na área da saúde. Foram excluídos os artigos que não se referiam à área da saúde ou que não mencionar pelo menos dois dos descritores utilizados.

Com o objetivo do melhor desenvolvimento do assunto na discussão do tema, os artigos foram divididos nos seguintes eixos temáticos: 1) Saúde: visando conhecer os relatos de experiência nas diferentes áreas da saúde que utilizam essa ferramenta; 2) Odontologia: visando conhecer os trabalhos na literatura desenvolvidos na área de Odontologia; 3) Atividade extracurricular: neces- 
sidade de estabelecer diferenças e confluências entre os termos atividades extracurriculares e ligas acadêmicas; e 4) Impacto na formação acadêmica: descrição sobre os aspectos pedagógicos da atividade e seu impacto na formação acadêmica.

\section{RESULTADOS}

Os resultados obtidos por meio do levantamento de dados nos bancos de informações científicas foram disponibilizados nas figuras 1 a 3 . Para todas as situações de agregação de descritores apresentadas, as bases de dados MEDLINE, PubMed e SciELO utilizaram a combinação dos termos em inglês. Porém, nas bases de dados LILACS e Google Scholar foram utilizados os mesmos termos na língua portuguesa, pois a inserção dos termos em inglês não gerou resultado. Sendo assim, os resultados dos itens encontrados foram somados e passaram a compor um único universo de termos.

Foram encontrados 3.552 artigos científicos e após a aplicação dos critérios de inclusão e exclusão permaneceram 30 documentos, os quais foram consultados integralmente sistematizados nos 4 eixos temáticos (tabela 1).

\section{DISCUSSÃO}

As LAs não são frequentes no meio acadêmico, apesar de existirem relatos de sua origem por volta do século vinte ${ }^{12}$. Contudo, seu uso tem crescido exponencialmente nos últimos anos, o que demonstra um interesse crescente, verificando-se um ganho significativo na produção científica abordando esse tema. Portanto, é essencial a busca constante pela compreensão de sua efetividade, influência e entender como as LAs são constituídas ${ }^{20}$.

A LA deve ser um espaço para troca de conhecimento mútuo e deve contribuir com a comunidade $^{21,22}$. A liga comumente se desenvolve pautada no princípio da tríade universitária: educação, pesquisa e extensão, contudo nem sempre esses três pontos são explorados.

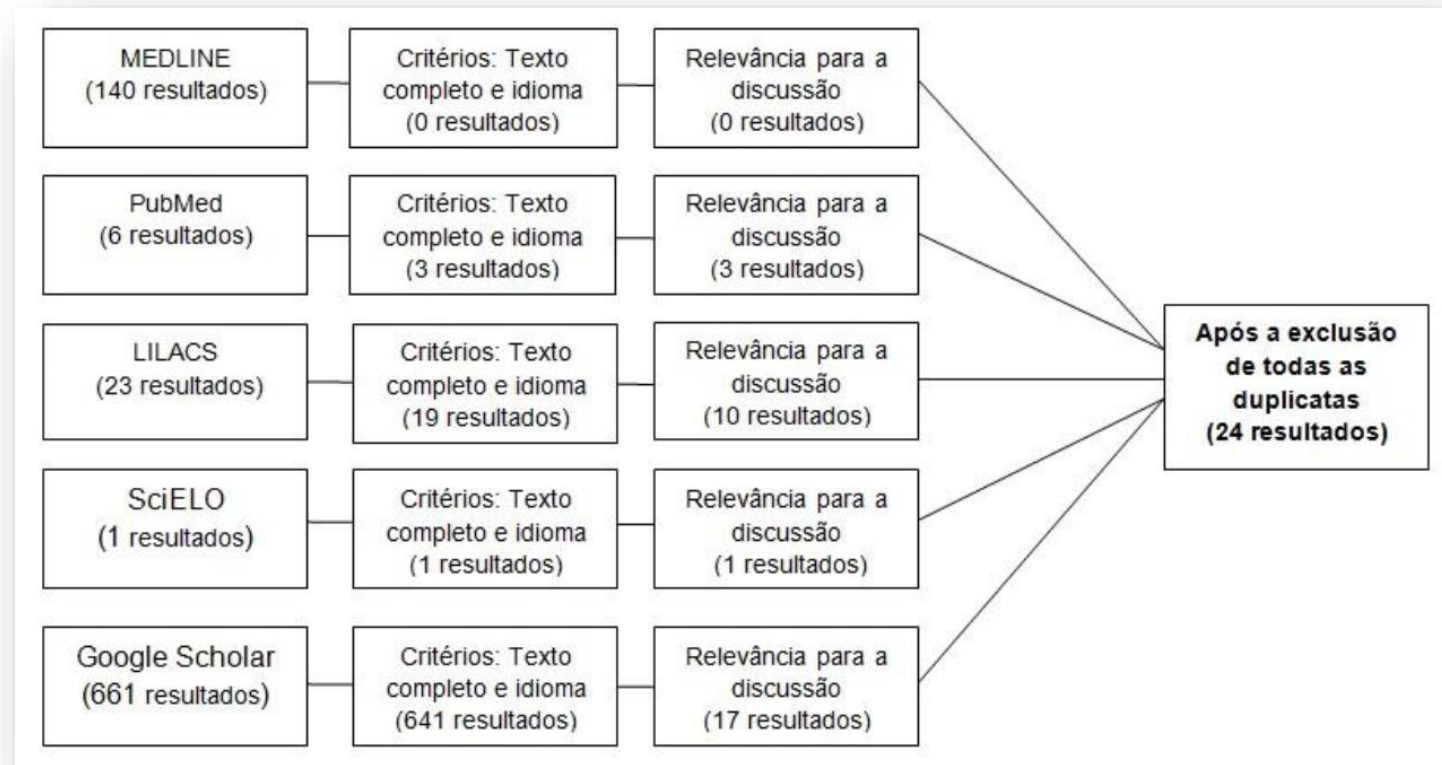

Figura 1. Fluxograma da pesquisa bibliográfica para a agregação de termos student league AND education 


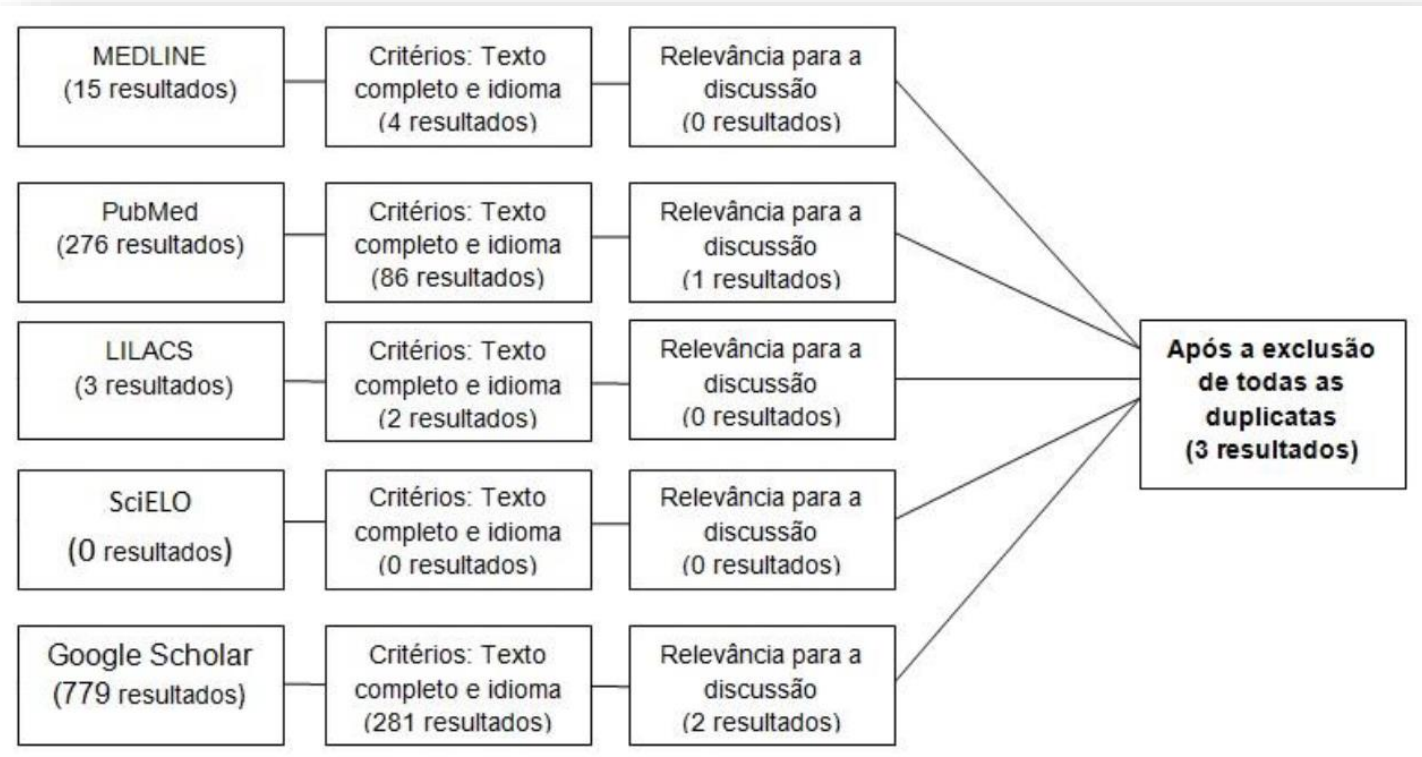

Figura 2. Fluxograma da pesquisa bibliográfica para a seguinte agregação de termos health education AND student league AND dentistry OR extracurricular activity

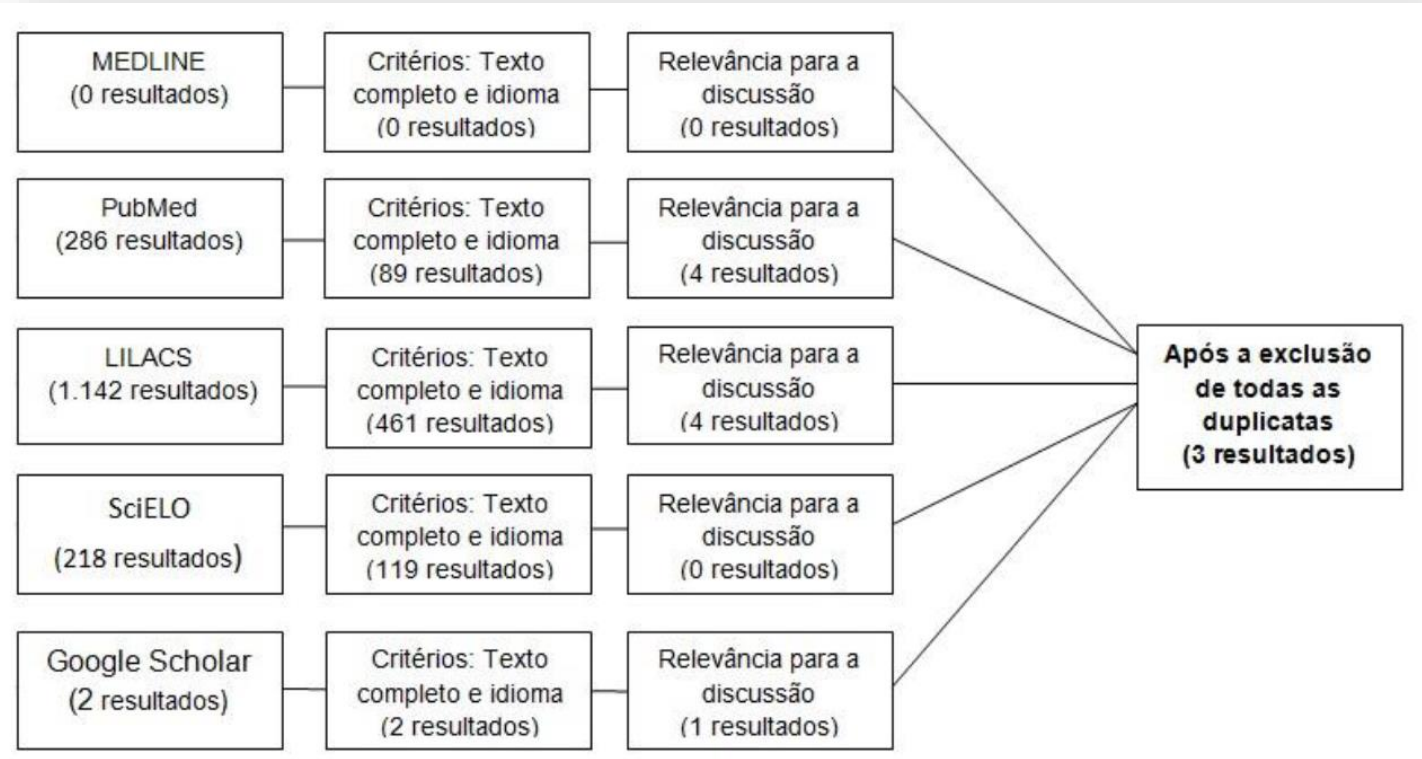

Figura 3. Fluxograma da pesquisa bibliográfica a seguinte agregação de termos education AND student leagues OR extracurricular activity 
Tabela 1. Sistematização dos artigos de acordo com os eixos temáticos

\begin{tabular}{lllll}
\hline Eixo temático & Estudo & Caracterização & Conclusão \\
\hline Saúde & Magalhães $(2015)^{23}$ & Discussão sobre a Com início através dos cursos \\
& Queiroz $(2014)^{30}$ & composição e impor- de Medicina, por volta da \\
& Floss $(2014)^{17}$ & tância e influência das década de 20, as LAs passaram \\
& Gonçalves $(2009)^{18}$ & LAs como instrumento a ser utilizadas por outras áreas \\
& Silva $(2009)^{24}$ & formativo para os da saúde como ferramenta \\
& graduandos de suas pedagógica. Hoje, encontram- & se relatos sobre ligas \\
& respectivas áreas. & acadêmicas de Psicologia, \\
& & Enfermagem, Odontologia \\
& & entre outras, além de \\
& & multidisciplinares. \\
\hline
\end{tabular}

\begin{tabular}{llll}
\hline Odontologia & Costa $(2015)^{16}$ & Aborda o tema prevenção & Apesar da carência de LAs
\end{tabular}
em saúde e expõem suas particularidades, além de descrever a experiência vivida na Liga Acadêmica Baiana de Educação em Saúde Bucal (LABESB).

\begin{tabular}{ll}
\hline Atividade & Dantas $(2017)^{5}$ \\
extracurricular & Bastos $(2012)^{9}$ \\
& Torres $(2008)^{26}$ \\
& Bonin $(2011)^{8}$ \\
& Andreola $(2016)^{10}$ \\
& Goergen $(2017)^{14}$
\end{tabular}

Discorrem sobre as
características das LAs, trabalho em equipe $\mathrm{e}$ importância na formação profissional bem como os processos de regulamentação e, atuação em comunidades pelos graduandos como promotores de saúde. odontológicas na literatura, é observa-se sua importância na formação dos estudantes de Odontologia.

As LAs possuem características que nos permite inferir que são atividades extracurriculares, como por exemplo, ocorrência em horário extracurricular, basear-se, também, em atividade de extensão e fornecer ao aluno experiências além das preconizadas no currículo formal.

\begin{tabular}{llll}
\hline $\begin{array}{l}\text { Impacto na } \\
\text { formação } \\
\text { acadêmica }\end{array}$ & Pêgo-Fernandes $(2011)^{26}$ & $\begin{array}{l}\text { Exposição de relatos de } \\
\text { experiência e dos recur- }\end{array}$ & Dentro da literatura, específica \\
& Hamamoto Filho & sobs, este eixo é \\
& $(2011)^{29}$ & sos pedagógicos que uma & amplamente abordado, eviden- \\
& Hamamoto Filho & liga pode oferecer para ciando o grande interesse em \\
& $(2010)^{28}$ & um aluno, pontuando entender de que forma as Ligas \\
& Silva $(2015)^{4}$ & fatores positivos e Acadêmicas contribuem para \\
& Monteiro $(2008)^{15}$ & eventuais fatores formação dos alunos, \\
& Fernandes $(2010)^{1}$ & negativos bem como os mensurando o nível de \\
& Imakuma $(2013)^{13}$ & motivos que levam a aprendizado e produção \\
& Soares $(2017)^{27}$ & procura dos graduandos cientifica atingida por parte \\
& Simões $(2014)^{22}$ & por esta atividade. & dos ligantes. \\
& Ramalho $(2012)^{11}$ & & \\
& Silva $(2015)^{4}$ & &
\end{tabular}

É notório, quando se apropria do tema, que essa ferramenta pode ser constituída de diferentes formatos. Existem ligas formadas por estudantes de um único curso, e existem ligas multidisciplinares.
Logo, podemos inferir que não existe um padrão para a sua formação, mas sabe-se que elas sempre são desenvolvidas por alunos e, comumente, possuem um professor orientador. 
As LAs são diferentes de acordo com seus objetivos, e estes são definidos conforme a necessidade do(s) estudante (s) envolvidos na sua criação. Algumas organizações acadêmicas priorizam suprir eventuais carências no currículo, como no caso da Liga de Oncologia e de Medicina Complementar e Integrativa (antes denominada Liga de Medicina Alternativa) da Universidade do Estado do Rio de Janeiro (UERJ), na qual os autores demonstraram a necessidade de aprofundamento neste tema e desenvolveram a LA ao invés de aguardar por uma alteração curricular administrativa ${ }^{17}$.

Existem também ligas que vão além da sala de aula, abordado atividades práticas em laboratórios e ações sociais, como é o caso da Liga Acadêmica Baiana de Educação em Saúde Bucal $(\mathrm{LABESB})^{16}$.

Para o acadêmico, as LAs trazem como benefício o contato precoce com os pacientes, vivência clínica e permuta de conhecimento por meio da relação com professores, profissionais da área e veteranos - logo, para um graduando iniciante, esta interface é desejável, trazendo para sua formação uma ampla experiência ${ }^{23,24}$.

Além do benefício acadêmico para os estudantes, as LAs trazem aspectos positivos às comunidades que são beneficiadas por meio de suas atividades. Muitas das vezes, essas ligas atuam em locais onde não há atendimento realizado por entidades públicas ${ }^{25}$.

São notórios os aspectos positivos das ligas, contudo existem autores que mencionam pontos negativos como sobrecarga das atividades curriculares e indução a uma especialização precoce $^{13,21,24}$.

Ao debruçarmos sobre a literatura consultada, podemos observar, sob o aspecto da saúde, que a grande maioria dos autores apresenta as LAs como um instrumento importante na formação durante a graduação, indicando uma crescente utilização desta ferramenta por diferentes áreas, muito embora na área de Odontologia, especificamente, ainda existam poucos relatos ${ }^{2,11,14-}$ 16,22 .

Do ponto de vista pedagógico e de impacto na formação acadêmica, nota-se o grande interesse em entender de que forma as LAs contribuem para a formação dos estudantes bem como a mensuração do nível de aprendizado e produção científica. Encontramos ainda sua caracterização e comparação a uma atividade extracurricular permitindo ao estudante experiências para além daquelas do currículo formal ${ }^{1,4,5,9,10,13,18,26,27}$.

Mesmo com todo seu potencial e impacto positivo na formação profissional, as LAs dependem também de uma organização administrativa e acadêmica para que regras e objetivos sejam cumpridos. Tal afirmativa pode ser corroborada através da experiência da Faculdade de Medicina de Botucatu (FMB) - Unesp, que em 2015 desenvolveu o Conselho das Ligas Acadêmicas (Conligac), estando este vinculado ao Centro Acadêmico, com intuito de estabelecer uma normatização para a abertura de ligas nesta faculdade, determinando parâmetros de planejamento e buscando um bom funcionamento das ligas, para então prover contribuições à formação médica ${ }^{28,29}$. Além deste relato, em 2006, foi criado a Associação Brasileira de Ligas Acadêmicas de Medicina (Ablam), durante $8^{\circ}$ Congresso Brasileiro de Clínica Médica, que contou com a colaboração de diversas entidades médicas ${ }^{30}$.

\section{CONCLUSÃO}

Conclui-se que as Ligas Acadêmicas, quando bem conduzidas por orientadores e bem organizadas, são benéficas para os graduandos. Elas são de fato atividades extracurriculares não obrigatórias e podem contribuir positivamente para o meio acadêmico e ainda apresentar relevância social. O estudante de Odontologia que busca desenvolver e compor uma liga acadêmica pode 
potencializar sua formação, tanto profissional quanto pessoal. As LAs são ferramentas pedagógicas úteis na formação do futuro profissional, mas necessitam de regras claras e bem definidas para que cumpram seu papel de forma plena.

\section{ABSTRACT \\ Academic Society of Dentistry: a literature review}

The Academic Society is defined as an extracurricular activity coordinated by students with mentoring and teacher participation, as well as the participation of researchers and health professionals. The members seek to contribute with their experiences, to explore and improve the student's knowledge about certain subjects. The current study aims to investigate the literature and understanding of the importance and contribution of Academic Societies for the development of dentistry pupils. The following key words were utilized as a search strategy: education, health education, extracurricular activities, academic societies and dentistry. The research was conducted in English, Portuguese and Spanish. The databases utilized were PubMed, Google Scholar, MEDLINE, LILACS and SciELO. A total of 3,552 published articles were found from 2000 to 2017. After applying the defined criteria, only thirty documents were selected and analyzed entirely. The results were arranged into the following four areas: Health, Dentistry, Extracurricular Activities and improvement of the academic curriculum. Based on the research, it was concluded that in spite of the extensive number of articles found, few addressed the topic of the Academic Society. The specific articles emphasized that Academic Societies are beneficial to their participants by improving self-learning, leadership, student relationships and the mutual exchange of knowledge between their participants and the public.

Descriptors: Education. Health Education. Dentistry.

\section{REFERÊNCIAS}

1. Fernandes FG, Hortêncio LO, Unterpertinger
FV, Waisberg DR, Pêgo-Fernandes PM, Jatene FB. Cardiothoracic Surgery League from University of São Paulo Medical School: twelve years in medical education experience. Rev Bras Cir Cardiovasc. 2010; 25(4):552-8.

2. Silva JH, Chiochetta LG, Oliveira LFT, Sousa VO. Implantação de uma Liga Acadêmica de Anatomia: desafios e conquistas. Rev Bras Educ Méd. 2015;39(2):310-5.

3. Van Vaisberg V, Cotrim FP, Ghaffar SA, de Andrade Hara LA, Monteiro RF, Tannuri U, et al. Operation and characteristics of the Academic League of Pediatric Surgery in quaternary center. Rev Méd. 2017;96(4):2414.

4. Silva SA, Flores O. Ligas Acadêmicas no processo de formação dos estudantes. Rev Bras Educ Méd. 2015;39(3):410-25.

5. Dantas AC, Santos MA, Gois MB. Importância da Liga Acadêmica para a formação profissional: aprendendo a trabalhar em equipe. International Nursing Congress. 2017;1(1):1-3.

6. Sardinha VS, Pires CA, dos Santos IC, Junior JM. A liga acadêmica na formação das equipes multiprofissionais: um relato de experiência. An Congr Bras Med Fam Comunidade, 2013; 12:1041.

7. Brasil. Resolução CNE/CES n ${ }^{\circ} 4$, de 7 de novembro de 2001. Institui Diretrizes Curriculares Nacionais do Curso de Graduação em Medicina. Diário Oficial da União. 09 Nov 2001; Seção 1:38.

8. Bonin JE, Oliveira JG, Nascimento JM, Rezende ME, Stopato SP, Leite IC. Liga Acadêmica de Medicina de Família e Comunidade: instrumento de complementação curricular. Rev APS. 2011; 14(1):50-7.

9. Bastos ML, Trajman AT, Teixeira EG, Selig L, Belo MT. O papel das ligas acadêmicas na formação profissional. J Bras Pneumol. 2012; 
38(6):803-5.

10. Andreola GM, Brenner FA. O papel das Ligas Acadêmicas de Dermatologia na formação acadêmica. Rev Méd UFPR. 2016;3(2):77-9.

11. Ramalho AS, Silva FD, Kronemberger TB, Pose RA, Torres MLA,Carmona MJC, et al. Anesthesiology teaching during undergraduation through an Academic League: what is the impact in students' learning? Rev Bras Anestesiol. 2012; 62:1:63-73

12. Botelho NM, Ferreira IG, Souza LE. Liga acadêmica de medicina: artigo de revisão. Rev Paraense Med. 2013; 27(4):85-8.

13. Imakuma ES. As Ligas Acadêmicas no ensino médico. Rev Medicina. 2013;92(4): 271-2.

14. Goergen DI, Hamamoto Filho PT. Lições aprendidas de um processo para regular a criação de Ligas Acadêmicas. Rev Ciênc Extensão. 2017;13(4):64-76.

15. Monteiro LL, Cunha MS, Oliveira WL, Bandeira NG, Menezes JV. Ligas acadêmicas: o que há de positivo? Experiência de implantação da Liga Baiana de Cirurgia Plástica. Rev Bras Cirurg Plástica. 2008; 23(3):158-61.

16. Costa RM, Silva JC, Santos MS, Figueiredo FM, Corrêa SP. Liga Acadêmica Baiana de Educação em Saúde Bucal (LABESB): experiência de discentes em Odontologia com educação em saúde bucal. Rev Bras Ciênc Saúde. 2015;17(3):219-26.

17. Floss M, Júnior AD, Teixeira TP. Liga de Educação em Saúde: Reflexões a partir das vivências dos estudantes de medicina da Universidade Federal de Rio Grande. Rev APS. 2014;17(1):116-119.

18. Gonçalves RJ, Ferreira EA, Gonalves GG, Lima MC, Ramos-Cerqueira AT, Kerr-Correa F, et al. Quem "Liga" para o Psiquismo na Escola Médica? A experiência da Liga de
Saúde Mental da FMB - Unesp. Rev Bras Educ Méd. 2009;33(2):298-306.

19. Ferreira DA, Aranha RN, Souza MH. Ligas Acadêmicas: uma proposta discente para ensino, pesquisa e extensão. Interagir. 2011;16:47-51.

20. Santana AC. Ligas acadêmicas estudantis. O médico e a realidade. Rev Medicina. 2012;45(1):96-8.

21. Silva AS, Rizzante FA, Picolini MM, Campos K, Corrêa CC, Franco EC, et al. Bauru School of Dentistry Tele-Health League: an educational strategy applied to research, teaching and extension among applications in tele-health. J Appl Oral Sci. 2011;19(6):599603.

22. Simões RL, Bermudes FA, Andrade HS, Barcelos FM, Rossoni BP, Miguel GP, et al. Trauma leagues: an alternative way to teach trauma surgery to medical students. Rev Col Bras Cir. 2014; 41(4): 297-302.

23. Magalhães EP, Rechtman R, Barreto V. A liga acadêmica como ferramenta da formação em Psicologia: experiência da LAPES. Psicol Esc Educ. 2015;19(1):135-41.

24. Silva HS, Galhardoni R, Fratezi FR, Almeida EB, Lima AM. Liga Acadêmica de Gerontologia da EACH/USP: histórico e perspectivas para a atuação do bacharel em Gerontologia. Kairós. 2009;12:131-41.

25. Torres AR, Oliveira GM, Yamamoto FM, Lima MC. Ligas Acadêmicas e formação médica: contribuições e desafios. Interface Comunic Saúde Educ. 2008;4(27):713-20.

26. Pêgo-Fernandes PM, Mariani AW. O ensino médico além da graduação: ligas acadêmicas. Diagn Tratamento. 2011;16(2):50-1.

27. Soares LR, Freitas-Junior R, Ribeiro LZ, Rahal RMS. Iniciação científica na graduação: experiência da Liga da Mama da Universidade Federal de Goiás. Rev Bras Mastol. 2017;27(1):21-5. 
28. Hamamoto Filho PT, Villas-Bôas PJ, Corrêa promoção de saúde. Frag Cultura. FG, Muñoz GO, Zaba M, Venditti VC, 2014;24:73-8. Schellini SA. Normatização da abertura de ligas acadêmicas: a experiência da Faculdade de Medicina de Botucatu. Rev Bras Educ Méd. 2010;34(1):160-7.

29. Hamamoto Filho PT. Ligas Acadêmicas: motivações e críticas a propósito de um repensar necessário. Rev Bras Educ Med. 2011;35(4):535-43.

\section{Correspondência para:}

Maria Isabel Souza

e-mail: profamariaisabel@yahoo.com.br

Núcleo de Teleodontologia UERJ

Boulevard 28 de Setembro, 157

Prédio Mário Franco Barroso, $2^{\circ}$. andar, sala 3

Vila Isabel

20551-030 Rio de Janeiro/RJ

30. Queiroz SJ, Azevedo RL. A importância das ligas acadêmicas na formação profissional e 